\title{
PRESENTACION DE LINEAS DE TRABAJO
}

\section{ESTLDIO DE UNA ZONA RURAL BAJO UN ENFOQUE INTERDISCI- PLINAR}

En el transcurso de los cinco últimos anos, hemos venido realizando un trabajo interdisciplinar con los alumnos y profesores de los diferentes Centros de Bachillerato donde se planteo esta actividad. Con ello, no hacemos más que seguir las directrices marcadas por la UNESCO en materia de Educacion Ambiental.

El enfoque interdisciplinar que se le da a este trabajo, implica la colaboractón de parte del profesorado, lo que conlleva una perfecta planificación, programación y organización de las correspondientes áreas educativas.

En la primera reunión de coordinación se trazarán las directrices a seguir durante rodo el curso academico: asignaruras que intervienen, zona de estudio, dctividades, etc. Posteriormente, cada profesor programa la disciplina teniendo presente los objetivos propuestos por todo el grupo.

Nosorros hemos realizado la interdisciplinaridad con las asignaturas del área de Ciencias (Geologia. Física, Quimica. Biologia, Matemáticas y Dibujo). Con el fin de poder valorar los objetivos propuestos, pasamos un test de entrada de conocimientos, donde planteamos una serie de cuestiones relacionadas con la temática ambiental. Asimismo, un test sociometrico para medir el nivel de relación que existe entre los diferentes grupos formados. Al finalizar el trabajo, se vuelven a pasar de nuevo los tests para medir las diferencias obteridas.

La verificacion de la actividad consiste en el adiestramiento por parte de los alumnos, en las técnicas necesarias para comprender la dinámica del entorno ambiental. Toda la programación consta primero de una parte teórica seguida de otra puramente práctica. La teoria suele ir acompanada de abundante material audiovisual, mientras que la parte práctica, se desglosa en laboratorio y de campo. La frecuencia de laboratorio es de una hora semanal y en ella se exponen de forma practica los conocimientos adquiridos en esos dias. Este aspecto se completa con una sali- da mensual al entomo del Centro Educativo, que nos sirve para tomar contacto directo con el medio natural. En la semana siguiente, se convoca una reunión conjunta de los diferentes grupos a fin de analizar y comentar las incidencias observadas.

Todo este trabajo se va desarrollando a lo largo del curso académico y en el entorno del propio alumnado. Ahora bien, para completar toda esta actividad, realizamos un campamento en una zona próxima. Con esto intentamos demostrar a nuestros alumnos que ya están preparados para verificar un trabajo serio de campo.

Pensamos que es de vital importancia la valoración que pueden dar los alumnos de todo el entramado didáctico que hemos expuesto. Por esta razón, también ellos evaluan las diversas partes de este trabajo, que nos servirá, ante to. do, para eliminar en años sucesivos los defectos observados.

ALVAREZ CALVO, I.A. I.N.B.A.D. Granada

CUBERO SANCHEZ, A. Departamento Historia, Facultad de Medicina, Granada

EN TORNO A UNA ACTUALIZA. CION DEL PROGRAMA DE PRAC. TICAS DE FISICA Y QUIMICA EN BACHILLERATO Y COU. APLICA. CION DE UNA EXPERIENCIA IN. DUSTRIAL.

La regulación del régimen de prácticas de laboratorio, como complemento indispensable de las asignaturas de Física y Química, es todavia hoy un tema pendiente de solución, en cuanto que su realización depende, en buena parte, de la iniciativa, esfuerzo y buena voluntad del profesorado. Se echa de menos una serte de incentivos tales como la posibilidad de dotar a los laboratorios de material no incluido en los lotes habituales, la existencia de publicaciones especializadas, en donde sea posible comunicar, comentar y promocionar trabajos y experiencias en este campo y, finalmente, la necesaria reestructuración de los grupos de alumnos a la hora de ocupar los puestos de labora- torio de manera que puedan ser atendidos eficazmente.

Otra cuestión ineludible es, a nuestro juicio, la necesidad de adecuar el tipo de prácticas, especialmente a nivel de COU, a temas de intimo contacto con la realidad industrial y tecnológica basados en las lecciones teóricas y como una aplicación de las mismas. A este efecto, y en uno de los cursos impartidos por nosotros en el I.B., "Luis Chamizon de Don Benito-Villanueva (Badajoz), se llevó a cabo la experiencia de instruir prácticamente a un grupo de alumnos en las tecnicas de análisis y control de calidad de las conservas vegetales producidas en la región, para lo que se contó con la ayuda incondicional de los fabricantes en el capitulo de material y productos especiales no disponibies en el Centro. Posteriormente en el I.B. "Valle de Jerte" de Plasencia (Cáceres), se repició, esta vez con pimentón, producto de gran importancia económica en la zona. En ambos casos los resultados han podido contrastarse como altamente satisfactorios ya que los alumnos no solamente consiguieron familiarizarse con una serie de tecnicas de trabajo comunes en el sector industrial, sino que también llegaron a integrarse dentro de su problemática general.

Creemos que todo esto puede ser un camino que ayude a mejorar y dar un sentido realista a la asignatura. En todo caso, es necesario, al menos, meditar sobre ello.

J. MARTINEZ GARRIDO. I.B. "Claudio Moyano», Zamora.

DIDACTICA ESPECIA L DE MATEMATICAS, FISICA Y QUIMICA. CURSO PARA LA OBTENCION DEL CERTIFICADO DE APTITUD PEDAGOGICA PARA FORMA. CION PROFESIONAL

Estas tineas tatan de plasmar un esquema del desarrollo y contenidos del curso. Dicho curso consta de treinta horas distribuidas en diez dias. Pasado dicho periodo, los Profesores-Alumnos (en su mayoria licenciados e ingenieros superiores) deberán realizar clases prác- 
ticas en los centros con ayuda del Pro fesor Tutor. El numero de ProfesoresAlumnos no debe pasar de treinta. El método de trabajo que se propone es el de dinámica de grupos y posterior puesta en común.

El contenido, por dias, es como sigue:

Día $1^{\circ}$. Estudio y análisis de los Cuestionarios Ofjciales de Matemáticas, Fisica y Quimica correspondientes al último Ciclo de E.G.B. Idem con los Cuestionarios Oficiales de Formación Profesional de Primer Grado (FP-I), orientaciones metodológicas y distribución horaria semanal.

Día $2^{\circ}$. Estudio y análisis de los Cuestionarios Oficiales de Matemáticas, Fisica y Quimica de Formación Profesional de Segundo Grado (FP-iI). Orientaciones metodológicas y distribución horaria semanal.

Día $3^{\circ}$. Programar una posible distribución de materia por evaluaciones con los cuestionarios de Matemáticas, Fisica y Química para una Rama de Formación Profesional. Discusión sobre el numero de evaluaciones a realizar durante un curso escolar.

Dia $4^{\circ}$. Programar un tema de Fisica de FP.I y otro de FP-II

Dia $5^{\circ}$. Programar un tema de Qui. mica de FP.I y otro de FP.Il.

Dia $6^{\circ}$. Programar un tema de $\mathrm{Ma}$ temáticas de FP-I y otro de FP-[1. Durante $\operatorname{los}$ dias 4,5 y 6 utilizar una ficha de programación para unificar criterios.

Día $7^{\circ}$. Metodologia para problemas. Metodología para Prácticas de laboratorio.

Dia $8^{\circ}$. Confeccionar una prueba sondeo inicial de Matemáticas, Fisica y Quimica con el objetivo de conocer el nivel de entrada en Formacion Profesional.

Dia $9^{\circ}$. Consideraciones sobre la programación de oposiciones.

Día $10^{\circ}$. Resumen de las conclusiones obtenidas durante el curso. Proyección de peliculas didácticas, diapositivas etc... Realización de una encuesta valorando distintos aspectos del curso.

Durante los dias del curso los Profesores-Alumnos utiłizaron diverso material como: fotocopias de cuestionarios oficiales de E.G.B., F.P., conclusiones de cursos y seminarios analogos, orientaciones metodológicas, cuestionarios y legislación de oposiciones, diversas programaciones de oposiciones, libros de texto de todos los niveles y cursos, libros de problemas, carpetas de Practicas de Laboratorio y fichas de programacion.

FRANCISCO HUESO UREÑA SEBASTIAN MURIEL GOMAR ICE de la Universidad je Córdoba

\section{VIDEOS I MICRORDINADORS PER ELS ESTUDIANTS DE QUIMICA DE LA UAB.}

Dintre d'una linea de renovació implicita en el món de l'ensenyament i a fi de que l'aprenentatge sigui el més agradable i efectiu possible, s'ha portat a terme a la Secció de Quimiques de la Universital Atatónoma de Barcelona una renovació de les classes de problemes i de práctiques que acompanyen al curs de Quimica General. Aquesta renovació, iniciada ja a l'octubre de 1983 . subvencionada per la Generalitat de Catalunya i amb colaboracio de l'ICE d'aquesta U.A.B., ha consistit fonamentalment en fer que les classes de laboratori siguin un «inici a la investiga- ción i no, en canvi, seguir una "recepta de cuinan i que les classes de problemes siguin una estona de raonament $i$ discussió i no una ucopia de la pissarras.

Per aconseguir aquestes fites s'han utilitzat mitjans d'aquesta decada: micrordinadors i videos. Actualment t'alumne ha de fer un simil de la pràctica en el micrordinador (aprox. 15 minuts) abans d'entrar al laboratori funes 15 sessions de 4 hores), on en els primers 10 minuts veu un video en el que s'explica, a base de primers plans, els detalls de la tecnica que haura de fer servir. Ambdues coses augmenten l'eficacia. disminueixen el cost humà de les practiques i permeten un canvi d'enfoncament de les mateixes.

A les classes de problemes no hi ha cap profesor a la pissarra. A l'hora de classe els alumnes resolen els problemes assignats uns dies abans $\mathrm{i}$ consulten els seus dubtes als professors. D'altre banda l'alumne té tots els temes teórics de! curs repartits en 30 hores de micrordinador on hi va voluntariament per a comprovar si el seu nivell de coneixements és l'adequat.

Una part del material utititzat correspon a una adaptació i traducció de programes i videos seleccionats d'entre molts existents i, concretament, procedeixen de la Universital de Michigan, Ann Arbor (Michigan) i de l'Editorial Wiley.

L'altra, ha estat feta per professors de la Secció de Quimica de la U.A.B. Un equip de varies persones ha treballat durant tot un any en aquest projecte que funciona amb gran acceptació per part dels estudiants des del començament de curs.

PILAR GONZALEZ DUARTE Cap de l'equip 